\title{
Adaptive 3D Rendering based on Region-of-Interest
}

\author{
Christel Chamaret ${ }^{\mathrm{a}}$, Sylvain Godeffroy ${ }^{\mathrm{a}}$, Patrick Lopez ${ }^{\mathrm{a}}$, Olivier Le Meur ${ }^{\mathrm{a}}$ \\ ${ }^{a}$ Thomson Corporate Research, 1 Avenue de Belle-Fontaine 35576 Cesson-Sevigne (France)
}

\begin{abstract}
3D processing techniques are really promising. However, several hurdles have to be overcome. In this paper, two of them are examined. The first is related to the high disparity management. It is currently not well mastered and its impact is strong for viewing 3D scene on stereoscopic screens. The second concerns the salient regions of the scene. These areas are commonly called Region-Of-Interest (RoI) in the image processing domain. The problem appears when there are more than one region-of-interest in a video scene. Indeed, it is then complicated for the eyes to scan them and especially if the depth difference between them is high. In this contribution, the 3D experience is improved by applying some effects related to RoIs. The shift between the two views is adaptively adjusted in order to have a null disparity on a given area in the scene. In the proposed approach, these areas are the visually interesting areas. A constant disparity on the salient areas improves the viewing experience over the video sequence.
\end{abstract}

Keywords: Visual attention, stereoscopic screen, 3D rendering, region-of-interest, adaptive blur...

\section{INTRODUCTION}

3D experiences have started for decades. 3D TV is expected to be the next revolution in the history of television.

However, the lack of effective visual comfort when watching a 3D content is one of the hurdles for 3D deployment. The main cause of visual discomfort is the accommodation-vergence mismatch [1]. While the viewer accommodates to the display, the eyes are verging to different depth planes according to the rendered scene content. This mismatch creates visual fatigue, and obviously, it is even increased when the objects in the scene are far out of the screen.

To mitigate this issue, some guidelines for filming in 3D have been defined [2]. Such rules prevent to have 3D objects very near to the viewer for a long while. Another principle consists in adapting the stereoscopic camera baseline, i.e. the distance between the camera optical axes, according to the depth of the scene. For instance, mechanically or by some processing operations, the baseline will be decreased in presence of object very near the camera.

Alternatively, these processing operations can be achieved no more at the content creation level but rather at the display level. Many stereoscopic players/displays offer the possibility to strengthen or not 3D aspects of the scene. It means that it is possible to put the scene into or out of the display. The 3D frustum is thus shifted, in order to better cope with the viewer fatigue. However, this shift is static and content agnostic. In that sense, it's far from being optimal.

In this paper, we propose a clever method to alleviate the visual discomfort by decreasing the accommodation-vergence mismatch in a dynamic way. For this purpose, the RoI of the scene is estimated, and the two views are adapted in such a way that the main RoI is displayed on the zero parallax plane.

In the section 2, we describe the whole post-processing chain inserted between the capture and the display, by describing precisely the way the RoI are estimated. Section 3 details how depth and RoI information are fused to produce an appropriate signal to modify the parallax. Section 4 will focus on temporal stability issues.

Then, the notion of additive blur for 3D rendering improvement will be described, and results will be provided in Section 6. 


\section{IMPLEMENTATION OVERVIEW}

\subsection{Context}

The proposed post-processing block uses as inputs some information derived from the stereoscopic views: the disparity map and the saliency map. Internally, the RoI is extracted and is merged with the disparity data. As output, the post-processing block provides stereoscopic views modified in such a way that the zero-parallax plane is adapted to the content.

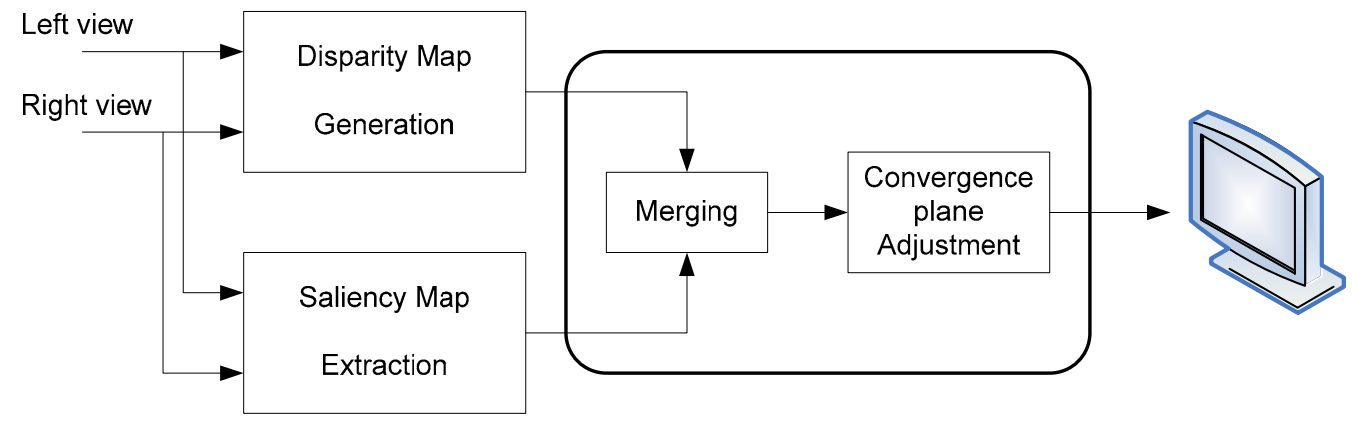

(a)

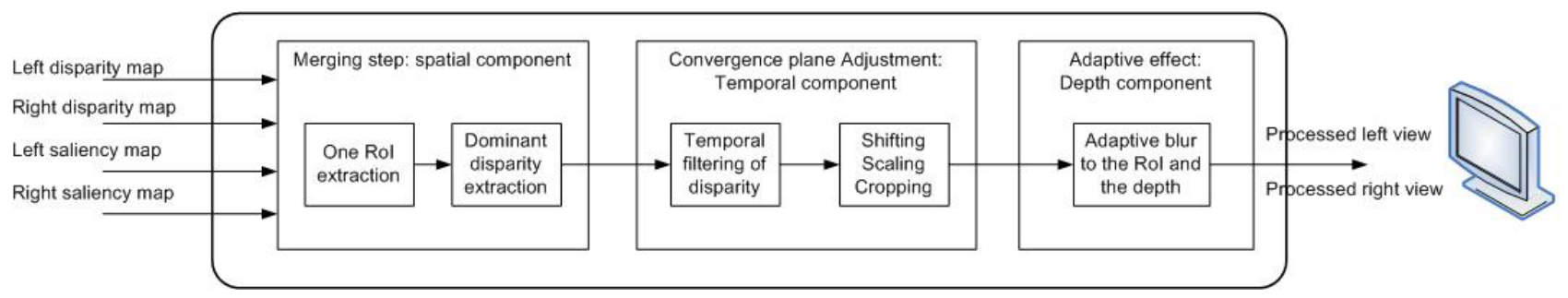

(b)

Figure 1. Overview block diagram: (a) Diagram of main principle. (b) Diagram with all steps described later in the sections.

\subsection{Disparity map}

On 3D stereoscopic displays, a same object is displayed on the left and the right views with a horizontal shift. This shift is commonly called parallax and its value depends on the depth of this object in the rendered scene. When this parallax is null, the object seems to be at the same depth as the display. Objects in front of (resp. behind) the display have positive (resp. negative) parallax values. The observer's brain fuses the two views and creates this 3D effect.

The disparity estimation block tries to determine for each pixel of each picture its displacement in the other picture. This displacement can be determined by well known block matching method [3][4]. However, such methods are usually not sufficient and additionally techniques based on belief-propagation or graph-cut may be added to improve the map quality [5]. In this paper, it is assumed that the disparity estimator block provides enough reliable maps in such a way that they can be further used for rendering purposes.

\subsection{Saliency map}

As our visual environment contains much more information than we are able to perceive at once, it is necessary to select the most important parts of a scene. This task is achieved by our visual attention. In other words, it guides the movement of the eyes, allowing an accurate inspection by the fovea of the chosen areas. Computational model of visual attention strives to detect the most salient parts of video sequences. Most of the models provide a topographic saliency sequence which quantitatively predicts how conspicuous every location in the input sequence is $\left(4^{\text {th }}\right.$ image from left-hand side in Figure 2).

These models are, for most of them, based on the use of the low-level visual features. These models are commonly called bottom-up models. At the opposite, top-down models take into account higher level of information as faces, text... or are dedicated to achieve a particular task. In Figure 2, a saliency map ( $4^{\text {th }}$ image from left-hand side $)$ is depicted for the current view. It highlights the most attractive regions. The low-level features include luminance, color contrasts and 
local motion of pixels. Indeed, objects moving in a video scene or objects with a color different from the background are likely to attract human visual attention.

In this contribution, the model described in [7], [8] is used. The information of luminance, color and motion information are merged to create a global saliency map called $S M_{i}$ ( $i$ indicates the frame number).

\subsection{Shifting/Cropping/Scaling}

The merging process has derived the disparity correction to be applied to each frame. This correction uniformly modifies the parallax of all pixels in the scene. It mainly consists in shifting the two views in opposite directions, in such a way that this added disparity matches the disparity correction notified by the merging process. However, a clipping must be added to the shift value. Indeed a negative disparity measured in $\mathrm{cm}$ on the display must be smaller than the inter-ocular distance. Otherwise, the eyes are constrained to diverge, which generates eye strain. Since this correction is not an integer number of pixels, this shifting is achieved by means of an interpolation filter.

A cropping is then applied on the shifted videos in order to discard the columns furthest to the left and the right, which are present in only one of the two views. Once this cropping has been performed, the number of columns of the resultant views is smaller than the original one. Thus, three main alternatives are possible:

- let the pictures as they are ;

- apply an horizontal scaling to fit the original resolution ;

- apply the horizontal scaling in both horizontal and vertical directions.

All solutions have their drawbacks, which are reduction of horizontal resolution, distortion of the picture and suppression of information in the top and bottom rows, respectively.

Since the shift parameter used is quite small compared to the horizontal resolution, , it has been decided to apply the second method. Visually, the distortion is small enough that it doesn't generate any annoyance to the observer.

\section{SPATIAL COMPONENT}

This step aims at extracting the disparity of the main region-of-interest in order to adequately tune the zero parallax plan at this disparity $d_{i}$ for each frame $i$. There are basically two tasks to accomplish in this step:

- The selection of a dominant region-of-interest out of a saliency map $S M$

- The extraction of an unique disparity value $d_{i}$ out of this spatial region-of-interest

\subsection{Region-of-interest extraction}

Several inputs are involved in the extraction of a unique region-of-interest. Although the saliency map provides crucial information on the interest of each pixel, additional information may be used to define a spatial area formalizing the main region-of-interest (cf. Figure 2). Then the $N$ most salient spots per frame as well as the depth map $D M$ are involved in this computation.

First, the pixels enclosed in the $N$ spots are set to be active (i.e. set to 255) in order to highlight $M$ regions. A region $R_{m, q}$ is defined by pixels which are considered as being adjacent, that means each pixel which is connected to at least one active neighbor (a neighbor being the eight pixels in the surrounding square). The size of the $m^{\text {th }}$ region in terms of pixels is defined by $q$. The biggest region, such as $R_{m, q_{-} \max }$, is retained as being probably the most important region-ofinterest. A binary map $R M$ is created out of this region, such as the pixels included in $R_{m, q_{-} \max }$ are set to 255 and the other one to 0 (second picture of Figure 2).

The selected region $R_{m, q_{-} \max }$ provides a coarse estimation of the main region-of-interest. This region is refined with additional information such as the depth and saliency maps, $D M$ and $S M$ respectively. A simple multiplication of each map combined to a thresholding process maintains the common important pixels in the final map $R M_{\text {refined }}$. 


$$
R M_{\text {refined }}(x, y)=\left\{\begin{array}{cl}
R M(x, y) \times S M(x, y) \times D M(x, y) & \text { if }>T_{0} \\
0 & \text { else }
\end{array}\right.
$$

Several iterations for choosing $T_{0}$ are done, such as the number of pixels superior to the threshold $T_{0}$ is at least $10 \%$ of q_max.
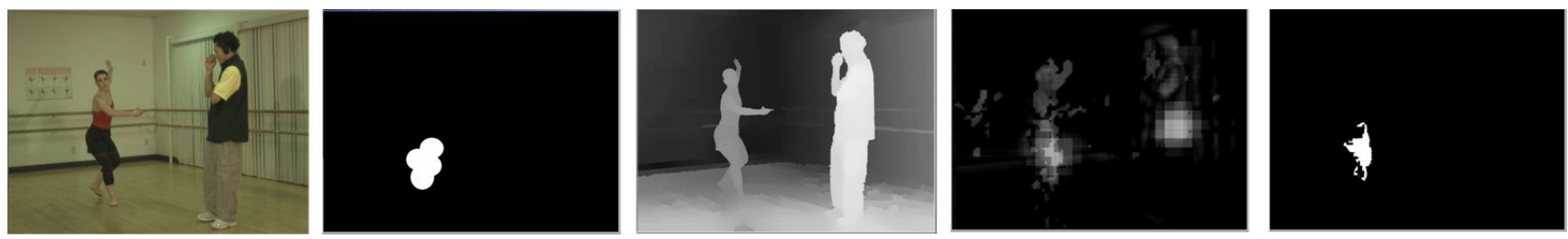

Figure 2. The different steps of the proposed approach for Ballet sequence. From left to right: the original frame, the map with the dominant region computed from the $N$ most salient spots, the depth map, the saliency map and the refined map providing the dominant region-of-interest.

\subsection{Matching the depth map}

Once the main region-of-interest $R_{\text {refined }}$ has been spatially marked within the refined map $R M_{\text {refined, }}$, a disparity value $d$ related to this area has to be found out of the depth map. However, several problems can be encountered. First, a spatial region may cover a range of disparity value. Second, some pixels of the background may be included in the main regionof-interest if the definition of $R_{\text {refined }}$ is not accurate enough.

To deal with these problem, a histogram (having a step of 5 unities of luminance) of all disparity values inside $R_{\text {refined }}$ is computed in order to estimate the spreading of disparity values. The dominant group $G_{q_{-} \max }$, that means having most of candidates, is selected. Then, a median filter is applied within this group in order to get out a reliable disparity value $d$, such as $d=\operatorname{Median}\left(G_{q_{-} \max }\right)$.

All the previously described processes are applied for each view independently. Therefore, for each region-of-interest $R_{\text {refined,view }}$ a disparity value $d_{\text {view }}$ is computed. Only at this stage, the redundancy induced by the two views is exploited in order to refine the data. The choice of disparity value between the two views is related to the one obtained at the previous frame $i-1$. The final disparity value for frame $i$ is the disparity value being the closest (regarding its absolute value) to the one of frame $i-1$. In other words, $d_{i}=d_{\text {view }, i}$ such as $\operatorname{Min}\left|d_{\text {view }, i}-d_{\text {view }, i-1}\right|$.

\section{TEMPORAL COMPONENT}

For each frame $i$, a disparity value $d_{i}$ related to the depth of the main region-of-interest is extracted. Due to potential discontinuities, it is recommended to smooth the disparity value $d_{i}$ temporally. In addition, the temporal smoothness should be calibrated adequately. On the one hand, the depth adaptation has to be fast enough for following change of RoI's depth or location and on the other hand, it should not be too fast to remain undetectable to any viewers. A special focus on the strength of the temporal filtering is provided in this paper.

\subsection{Smooth the depth}

The depth map may have a poor quality leading to strong discontinuities between the depths extracted from frame to frame. Additionally, the determination of the main region-of-interest location may be wrong or noisy. The depth value is then temporally smoothed.

Kalman filter has been described many times in literature [6]. The principle is based on finding the solution of the state and measurement equations (more details in [6]). Knowing that only the disparity has to be smoothed, the equations are expressed for one dimension:

$\hat{\bar{x}}_{k}=a \cdot \hat{x}_{k-1}+u_{k-1}$ is the state equation and $z_{k}=h \cdot x_{k}+v_{k}$ is the measurement equation.

$v$ and $u$ are the process and the measurement noise respectively. The state does not change from step to step, then $a=1$. The noisy measurement is considered constant, then $h=1 . \hat{\bar{x}}_{k}$ is the a priori state, it is estimated at step $k$ given the knowledge of the process prior to step $k$ (before the injection of the residual, that is the difference between the predicted 
measurement and the actual measurement). $\hat{x}_{k}$ is the a posteriori state, estimated at step $k$ given the measurement $z_{k}$. Let us define the a priori and a posteriori estimate errors, such as: $\bar{e}_{k} \equiv x_{k}-\hat{\bar{x}}_{k}$ and $e_{k} \equiv x_{k}-\hat{x}_{k}$. The corresponding a priori and a posteriori estimate error covariances are: $P_{k}^{-}=E\left[\bar{e}_{k} \cdot \bar{e}_{k}^{T}\right\rfloor \quad$ and $P_{k}=E\left[e_{k} \cdot e_{k}^{T}\right\rfloor$

In deriving the kalman filter equations, the a posteriori state is given by: $\hat{x}_{k}=\hat{\bar{x}}_{k}+K \cdot\left(z_{k}-\hat{\bar{x}}_{k}\right)$. The difference $\left(z_{k}-\hat{\bar{x}}_{k}\right)$ is the residual. $K$ is chosen to be the Kalman gain that minimizes the a posteriori error covariance. One form of the resulting gain $K$ is: $K_{k}=\frac{P_{k}^{-}}{P_{k}^{-}+R}$. The associated noise covariance of $u$ and $v$ are given by $R$ and $Q$.

A typical implementation of the Kalman algorithm is given below. There are three steps.

Initial state:

$\hat{x}_{0}=C_{0} ; P_{0}=E\left[e_{0} \cdot e_{0}^{T}\right] \approx 0$ avec $e_{0} \equiv x_{0}-\hat{x}_{0} \approx 0$

Time update:

1) Project the state ahead $\hat{\bar{x}}_{k}=\hat{x}_{k-1}$

2) Project the error covariance ahead $P_{k}^{-}=P_{k-1}+Q$

Correction, measurement update:

1) Compute the Kalman gain: $K_{k}=\frac{P_{k}^{-}}{P_{k}^{-}+R}$

2) Update estimate with measurement: $\hat{x}_{k}=\hat{\bar{x}}_{k}+K_{k} \cdot\left(z_{k}-\hat{\bar{x}}_{k}\right)=\hat{x}_{k-1}+\frac{P_{k}^{-}}{P_{k}^{-}+R} \cdot\left(z_{k}-\hat{x}_{k-1}\right)$

$z_{k}$ being the measurement value at step $k$, it corresponds to the disparity $d_{i}$ extracted at frame $i$. Finally, one can express:

$$
\hat{x}_{k}=\hat{x}_{k-1}+\frac{P_{k}^{-}}{P_{k}^{-}+R} \cdot\left(d_{k}-\hat{x}_{k-1}\right)
$$

3) Update the error covariance: $P_{k}=\left(1-K_{k}\right) \cdot P_{k}^{-}$

$P_{k}=\left(1-\frac{P_{k}^{-}}{P_{k}^{-}+R}\right) \cdot P_{k}^{-}=\left(1-\frac{P_{k-1}+Q}{P_{k-1}+Q+R}\right) \cdot\left(P_{k-1}+Q\right)$

Presuming a very small process variance, $Q$ is assumed to be close to 0 . Most of systems which use a Kalman filtering for smoothing trajectory set the different noises $R$ and $Q$ to a constant value. The quantity of noise applied to a system determines the strength of the smoothness. In a first time, $R$ has been set empirically to 40 .

\subsection{Adequate measurement noise $R$}

In a first approximation, $R$ has been set to coherent constant value in order to smooth the disparity value frame to frame. Nevertheless, one can imagine there is a maximal skip of shift that may be applied to the two views after which the skip is detectable by any observers. User tests have been conducted in section 6.2 in order to determine this maximum shift difference $\Delta s_{\text {user }}$ (or $\Delta d_{\text {user }}$ if speaking about disparity) having in mind the idea of Just Noticeable Difference. These results will design the parameters of Kalman filtering. 
In addition to the maximal shift value, a difficult point is the range of disparity which is intrinsic to the sequence. A large range of disparity for a sequence provides a higher scale for the shift value. The strength of the temporal filtering has to be linked to the disparity range of the sequence.

Let us assume $R$ is changing over disparity range defined by the considered sequence, that means $R$ changes as a function of the minimal and maximal disparities, such as $R_{D}=\mathrm{f}\left(d_{\min }, d_{\max }\right)$ and $D=d_{\max }-d_{\min } . Q$ is assumed being close to 0 .

From previous usual Kalman algorithm and knowing the constraint on maximum shift $\left(\hat{x}_{k}-\hat{x}_{k-1} \leq d_{u s e r}\right)$ between previous estimated and current predicted value, a new algorithm may be defined to find the estimate of $R_{D}$ for the current sequence:

$$
\begin{aligned}
& \text { Initial state: } \\
& \hat{x}_{0}=C_{0} ; P_{0} \approx 1 \\
& \text { Update estimate with measurement: } \\
& \text { The estimate state is now computed considering } R_{D} \text {, then } \hat{x}_{k}-\hat{x}_{k-1}=\frac{P_{k}^{-}}{P_{k}^{-}+R_{D}} \cdot\left(z_{k}-\hat{x}_{k-1}\right) . \\
& \text { With } \hat{x}_{k}-\hat{x}_{k-1}=d_{\text {user }}=P_{k-1} \cdot\left(\frac{z_{k}-\hat{x}_{k-1}}{d_{u s e r}}-1\right) \\
& \text { Update the error covariance: } \\
& \qquad P_{k}=\left(1-\frac{P_{k-1}}{P_{k-1}+R_{D}}\right) \cdot P_{k-1}
\end{aligned}
$$

In section 6.2, these equations are exploited with the user tests.

\section{DEPTH COMPONENT}

A third component has been involved in the rendering of region-of-interest: the dimension related to the depth. In order to create an interesting visual effect, an adaptive blur has been introduced as a function of the depth and the degree of interest of the pixel. In order to limit the eye strain due to high disparity ranges, this adaptive blur function plays the role of a guide to the attention.

The depth of the main region-of-interest is considered as being the reference $d_{R O I}$. All pixels of the two views including the main RoI itself which are located at this same disparity are not blurred. Then, the depth difference, $d_{\text {distance }}$ for the current pixel $p_{-}$current, such as $d_{d i s \tan c e}=\sqrt{\left(d_{R O I}-d_{p_{-} \text {current }}\right)^{2}}$, defines the quantity of blur to apply to the current pixel $p_{-}$current. The higher the depth difference is, the stronger the blur is applied.

The quantity of blur is controlled by the kernel size of an average filter, such has size $=\frac{d_{\text {dis } \tan c e}}{d_{\max }} \cdot C+\ln \left(\frac{d_{\text {dis } \tan c e}}{d_{\max }} \cdot C\right)$ where $d_{\max }$ is the maximal available distance.

$C$ is the blur coefficient and is set by the user; it controls the global quantity of blur. The average filter kernel is $\frac{1}{9} *\left[\begin{array}{lll}1 & 1 & 1 \\ 1 & 1 & 1 \\ 1 & 1 & 1\end{array}\right]$. Five structuring elements are defined having a size in the following set $\{3,5,7,9,11\}$. Some visual results are depicted in the next section. 


\section{VALIDATION OF RESULTS}

This section is related to the experimental validation of the proposed approach.

The first section deals with the validation of the spatial dimension; basically it concerns the validation of the detection of the main region-of-interest. The second section is related to the temporal filtering and the third section is a visual validation of 3D sequences.

\subsection{Validation of region-of-interest detection}

Rather than a quantitative measure of validation for the detection of region-of-interest, a visual assessment has been performed on each available sequence. As observed in Figure 3, the airplane is detected as being salient by the visual attention model (second row, bright areas indicate the most visually important regions). Then, the detection of a unique spatial region for extracting a global depth is well performed, as being depicted by the green pixels (third row).

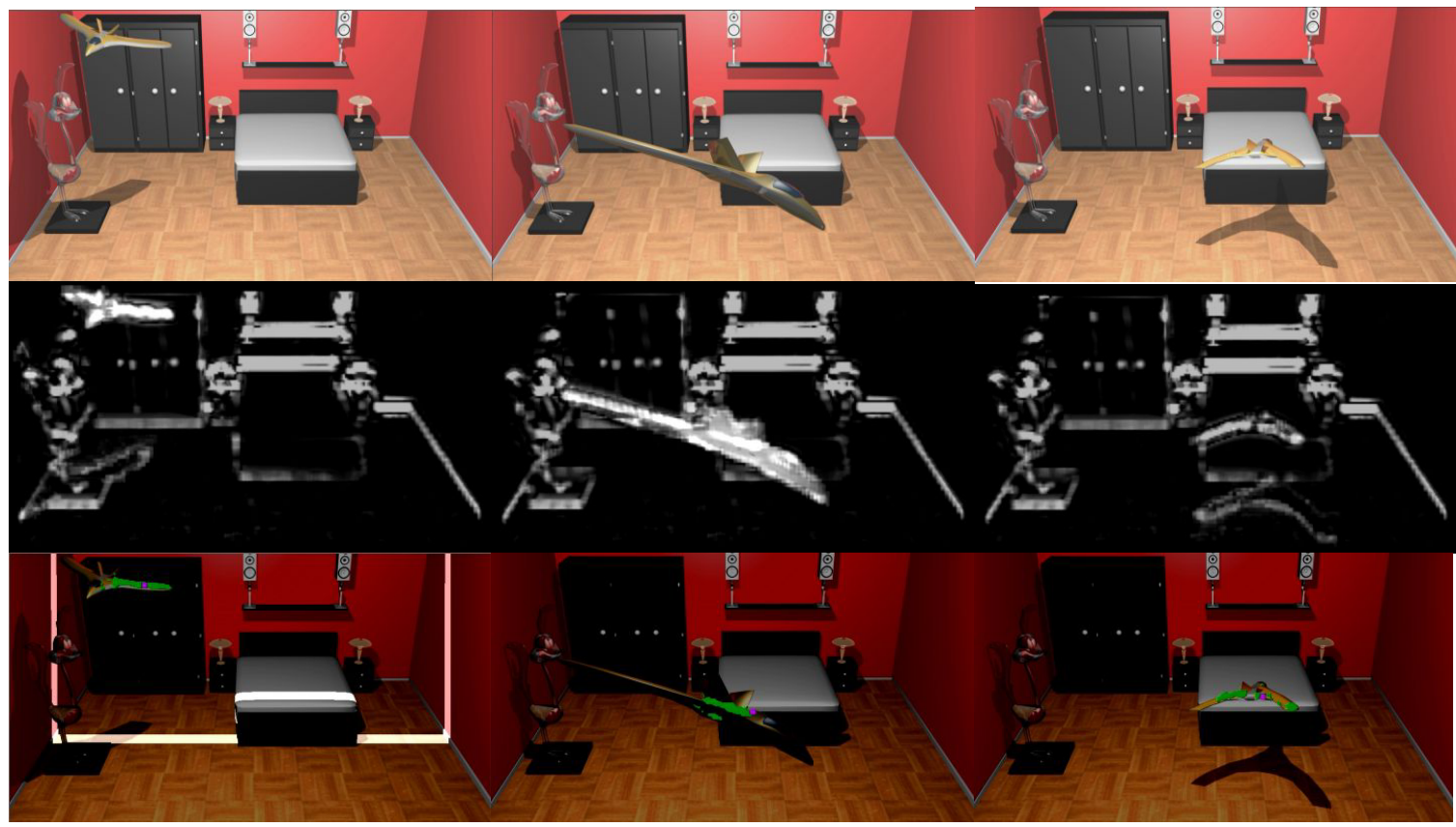

Figure 3. Top raw is the original frame of sequence airplane for frames 47, 112 and 249. Second raw is the corresponding saliency maps. Third raw allows a visual validation: green pixels are the main detected region-of-interest; bright pixels depict the current convergence plan. The convergence plan is visible only for the frame 47; regarding the frame 112 it is in front of the scene and regarding the frame 249 , the zero parallax plan had no enough time due to temporal filtering to catch up with the airplane plan yet.

More information concerning the performance of the computational model of visual attention can be found in [7], [8].

\subsection{User tests to tune the temporal filtering}

The idea behind this user tests is to determine the maximum value of shift that one can apply between two views without any notification coming from the observers (Just Noticeable Difference). A protocol with several sequences and patterns of shift changes has been defined.

\section{Protocol of user tests}

Two different contents have been used to create the two sequences:

- LoveBirds sequence: two people are walking, going closer to the observer. A house as well as a tree depicts the background.

- LapTop sequence: two people sit down and stand up to shake hands. 
A still scene has been created out of each sequence (one frame has been copied for several seconds) because motion of a scene may potentially hide the shift variation. Then, the worst case is considered for detecting the shift changes.

Several patterns of shift evolution have been defined in order to double-check user remarks; they are presented in Figure 4:

- Progressive Steps: steps are becoming higher and higher over time.

- Progressive skips: steps are becoming higher and higher over time, but the shift value come back to the original value. This pattern may avoid some effect due to the content as may happen with previous pattern.

- Random Shifts for sequence LoveBirds: The shift evolution is random.

- Random Shifts for sequence LapTop: The shift evolution is random.

The shift changes are varying as defined in the patterns and the observer has to detect a skip between views. A typical shift skip is showed at the beginning of the test in order to get him familiar with the annoying effect to detect. Observers have the possibility to view several times the video sequence.

Pattern1: Progressive Steps

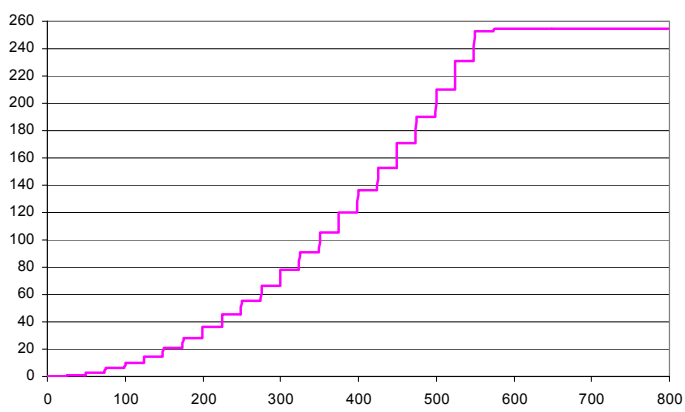

Pattern3(a): Random Shifts for sequence LoveBirds

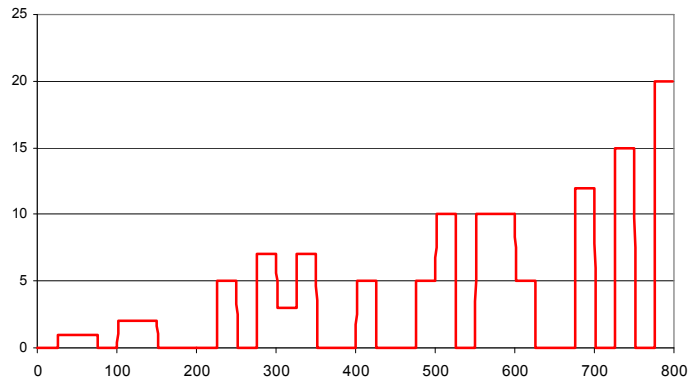

Pattern2 : Progressive Skips

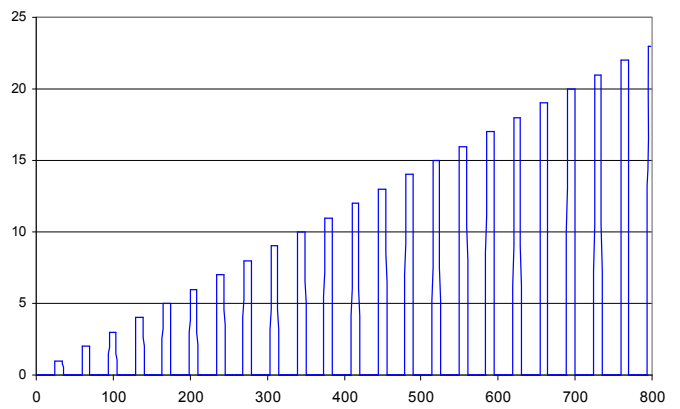

Pattern3(b): Random Shifts for sequence LapTop

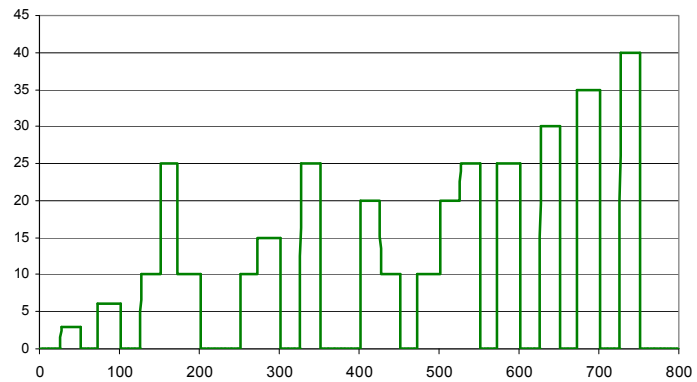

Figure 4. Patterns of shift changes applied to two views.

The observers are invited to focus on the same region-of-interest. The Miracube (G460X) screen is used for the test and observers are located two meters away from the screen. Twenty three observers performed the test.

\section{Maximal shift value or Just Noticeable Difference of shift contrast}

The results for all users are averaged and are given in Figure 5 per sequence and pattern. Finally, the average of all sequences is computed in order to get a final maximal value: $\Delta s_{\text {user }}=1.5$. The pattern of random laptop was a posteriori not thin enough. If excluded from the final average, the $\Delta s_{\text {user }}$ would be equal to 1.41. Since the influence of 
this pattern was not so high and that it seems not to be appropriated to this experiment, the maximal shift value is kept, i.e. $\Delta s_{u s e r}=1.5$.

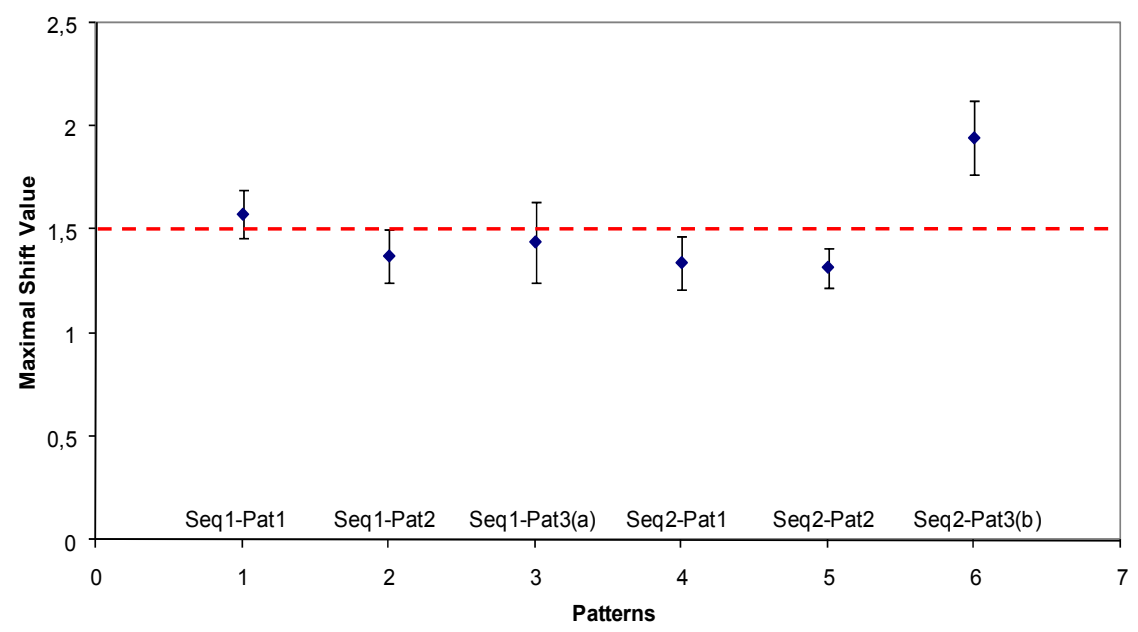

Figure 5. Maximal shift value extracted from the user tests per sequence and per pattern. Red line is the average value computed from the 6 patterns. Blue point is the measured shift value averaged over all observers per sequence and pattern. The standard deviation around this value is presented as well.

\section{Optimal smooth of the shift parameter}

As determined previously with a set of observers, the maximal shift increase to apply without any visual notification is 1.5; it corresponds to a difference of shift amplitude. The relationship between the shift and the disparity is mentioned in equation (4); if using a shift difference, the new relationship is mentioned in (5), such as $\Delta s_{\text {user }}=s h i f t-d_{\min }=1.5$ :

$$
\text { depth }=255 \cdot\left(\frac{\text { shift }-d_{\text {min }}}{d_{\max }-d_{\min }}\right)(4) \quad \Delta \text { depth }_{\text {user }}=255 \cdot\left(\frac{\Delta s_{\text {user }}}{d_{\text {max }}-d_{\text {min }}}\right)(5)
$$

Finally, when substituting this equation into equation (3), the final expression of the measurement noise covariance $R_{D}$ as a function of the maximal and minimal disparities of a sequence is:

$$
R_{D}=P_{k-1} \cdot\left(\frac{255 \cdot\left(\frac{\Delta s_{k}}{d_{\text {max }}-d_{\text {min }}}\right)-\hat{x}_{k-1}}{255 \cdot\left(\frac{\Delta s_{u s e r}}{d_{\text {max }}-d_{\text {min }}}\right)}-1\right)
$$

The idea of this section is to find a typical behavior to apply to $R_{D}$ depending on the disparity range of the input sequence. Five sequences with different disparity ranges were available to determine with the previous equation and experimentally the optimal $R_{D}$.

Considering a set of input patterns $p$ rather than reasoning on time $k, R_{D}$ value is highly influenced by the previous inputs $\hat{x}_{k-1}$, so by the pattern. Let us consider the worst case, such as a variation from 0 to 255 . The number of 0 before the variation to 255 creates several patterns for which the system interacts differently. The patterns are defined like $\hat{x}_{0 \ldots M}=\{0 \ldots 255\}$ such as $\hat{x}_{M}=255$ and $\hat{x}_{0 \ldots M-1}=\{0\}$. These patterns represent the worst cases and Figure 6(a) depicts the variation of measurement noise as a function of the disparity range for different patterns. The two extreme cases are introduced; in order to find the intermediary case, a set of five sequences with different disparity range and their own pattern of shift variation without temporal filtering have been used to interpolate the intermediary curve (red 
curve in Figure 6 (a)). Then, this curve has been separated into two parts to determine a model (Figure 6 (b)): a slope equal to 0 till a disparity range inferior to 20 and a complex slope. Finally, one can define the following rule as drawn in (b):

$$
R_{D}= \begin{cases}40 & \text { if } \operatorname{abs}\left(d_{\text {max }}-d_{\text {min }}\right)<20 \\ 5.85 \cdot \operatorname{abs}\left(d_{\text {max }}-d_{\text {min }}\right)-77.3 & \text { else }\end{cases}
$$

One can notice that the empirical value of noise (section 4.1) was 40 , which is clearly a coherent part of this curve.

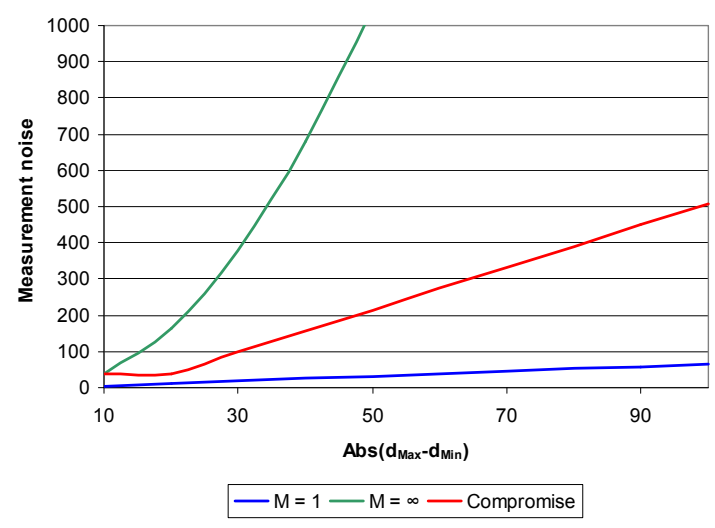

(a)

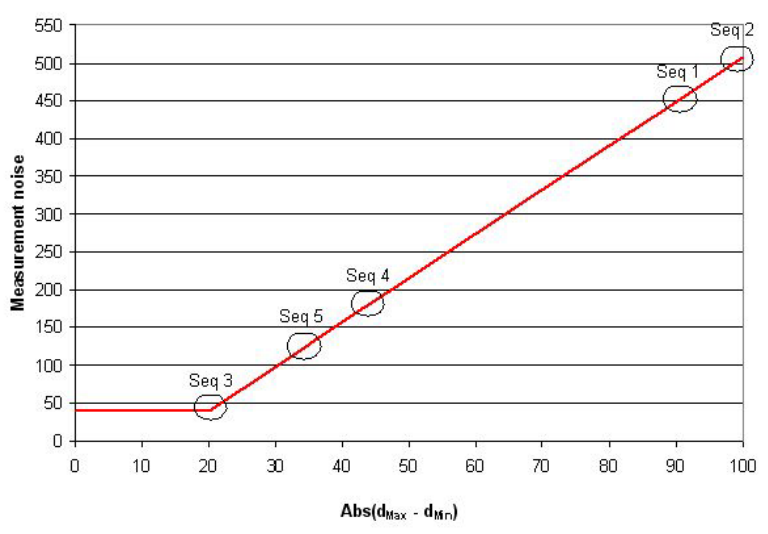

(b)

Figure 6. (a) Variation of measurement noise as a function of disparity range for several patterns. The slope may vary significantly. (b) Model of the variation of measurement noise as a function of disparity range for a set of sequences.

Figure 7 presents the filtered depth as a function of the value of measurement noise. The empirical noise value initially defined to 40 leads to a light smoothness of depth, probably not strong enough to avoid the visual detection of shift skip.

\section{Temporal filtering of extracted depth}

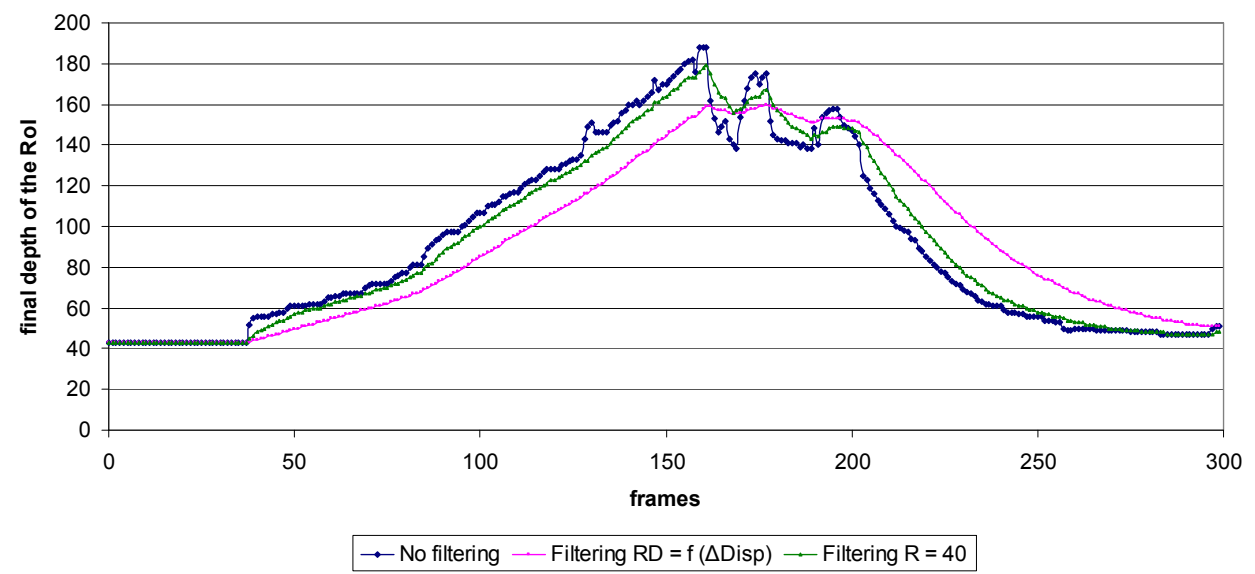

Figure 7. Variation of estimated depth after temporal filtering as a function of time for several values of noise measurement.

\subsection{Visual experiments}

Regarding the adaptation of region-of-interest at the zero parallax, most of people could not see any temporal changes. Few people were a bit annoyed not by the shift changes but by the scaling process which may be perceptible. Figure 8 shows the two visual results for two views when adapting the convergence plan. The results are more pleasant to watch due to the fact that the earth is not popping out anymore at a high disparity. The $3 \mathrm{D}$ effect is confined inside the screen. 
Figure 9 presents the results for the adaptation of convergence plan combined with the adaptive blur effect. The adaptive blur helps the convergence process on the region-of-interest, because it guides the attention. The user has less freedom to look around. Blur effect is quite natural, because its intensity is based on the depth of the focus object.
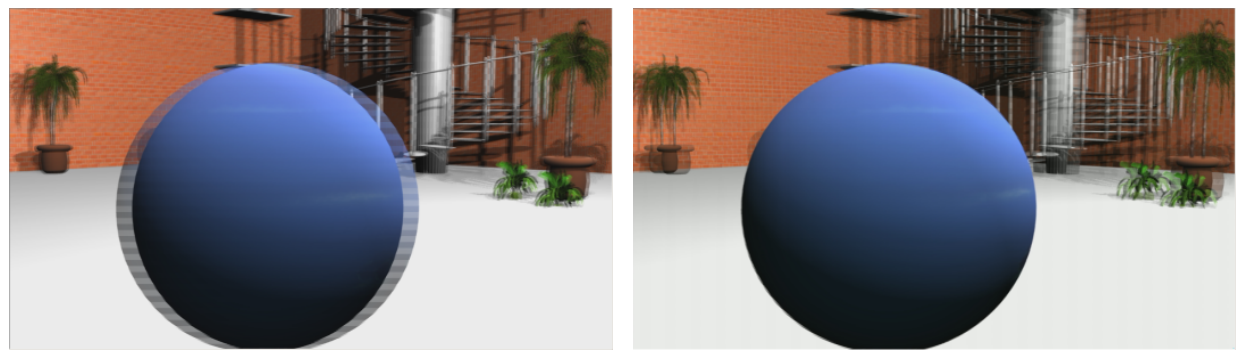

Figure 8. Influence of adapting the convergence plan at the RoI. Left-hand side is the original sequence with the ball being out of the screen originally. Right-hand side the zero parallax is located on the ball plan (RoI)
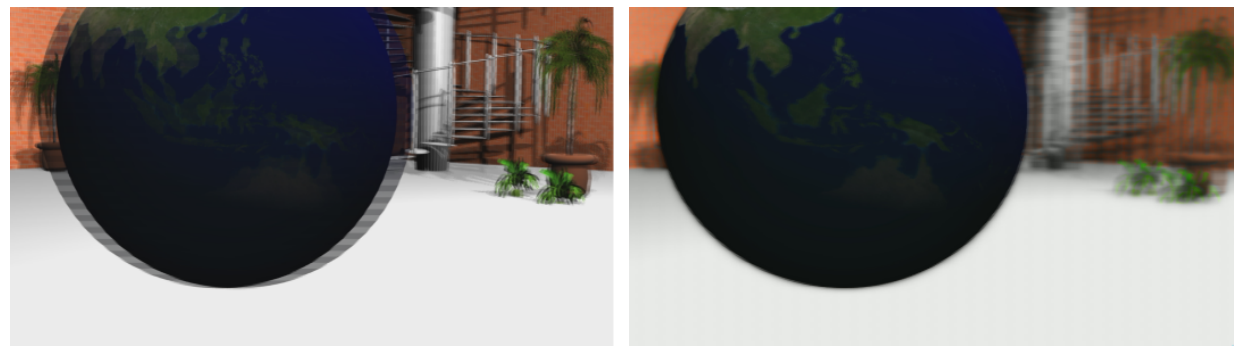

Figure 9. Visual rendering of adaptive blur. Left-hand side is the original frame; right-hand side is the blurred frame.

\section{CONCLUSION}

The introduction of 3D-TV is becoming increasingly feasible because of recent technologies and breakthroughs in image processing, display design and camera development as well as an improved understanding of 3D human factors. However, several problems remain. The most important one is likely the visual experience. The visual experience, or the quality of experience, is composed of a number of items such as perceived image quality, perceived depth and visual comfort.

In this study, we proposed to improve these three points by using a computational model of visual attention. The idea is to favor the quality and the visual comfort over the region that will unconsciously attract our gaze. One solution to deal with that is to adapt dynamically the shift between the two views in order to ensure a constant disparity on the region-of -interest.

Another contribution of this study concerns the psycho-physic user tests. This experiment estimated the just noticeable difference of a contrast of depth. The conclusion of tests allows us to tune accurately the different filters used in the proposed approach. However, it is required to pursue these tests with more video sequences, with more patterns of test... in order to be more robust.

Finally, our future work will deal with these kinds of experiment. In addition, it would be important to assess the ability of a computation model of visual attention, initially designed for $2 \mathrm{D}$ video sequences, to predict region-of-interest in a $3 \mathrm{D}$ context.

\section{REFERENCES}

[1] Hoffman D.M., Girshick A.R., Akeley K, Banks M.S., "Vergence-accommodation conflicts hinder visual performance and cause visual fatigue", Journal of Vision, Volume 8, Number 3 (2008)

[2] Wittlief K, "Stereoscopic 3D Film and Animation - Getting It Right", Computer Graphics, Vol 1, n³ (2007).

[3] Cheng, J. and Feng, S., "A real-time multiresolutional stereo matching algorithm", Proceedings of ICIP (2005) 
[4] Sharstein D. and Szeliski R., "A taxonomy and evaluation of dense two frame stereo correspondence algorithms", IJCV, Volume 47, (2002)

[5] Penc J. and al, "Graph-Cut and Belief-Propagation Stereo on Real-World Image Sequences", MI Tech report (2009)

[6] Welch G., Bishop G., "An Introduction to the Kalman Filter”, University of North Carolina at Chapel Hill, 1995.

[7] Le Meur O., Le Callet P., Barba D., Thoreau D., "A coherent computational approach to model bottom-up visual attention". IEEE Trans. On Pattern Analysis and Machine Intelligence, 5(28), 2006.

[8] Le Meur O., Le Callet P., Barba D., "Predicting visual fixations on video based on low-level visual features". Vision Research, 47(19):2483-2498, 2007. 